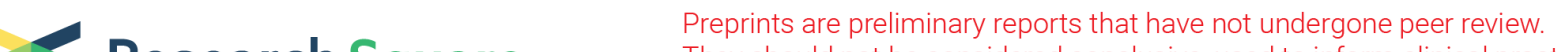 Research Square They should not be considered conclusive, used to inform clinical practice, or referenced by the media as validated information.
}

\section{An Arduino Real-Time Jump Tagging System for Exploring Stereotyped Jumping Behavior in Mice}

Junye Ge

Shaanxi Normal University

Guangyuan Li

Wuhan University of Technology

Haibo Zhang

Shaanxi Normal University

Haiying Liu

Fourth Military Medical University

Chuchu Qi

Fourth Military Medical University

Yuke Lin

Hangzhou Medical College

Qian Xue

Fourth Military Medical University

Shengxi Wu

Fourth Military Medical University

Yihui Liu

Shaanxi Normal University

Wenting Wang ( $\square$ wwt0657@fmmu.edu.cn)

Fourth Military Medical University https://orcid.org/0000-0002-8344-0102

\section{Methodology}

Keywords: Arduino, Jump, Stereotyped behavior, Ca2+ signal, Mouse

Posted Date: June 16th, 2021

DOI: https://doi.org/10.21203/rs.3.rs-603428/v1

License: (c) (i) This work is licensed under a Creative Commons Attribution 4.0 International License. Read Full License 


\section{Abstract}

The jump is one of the common stereotyped behavior in rodents. It is the natural state in some types of mice and also can be found in certain types of disease models, such as addiction. It is straightforward and easy to identify by the human eye in offline analysis. However, jumping is a short-lived act that happens immediately. It is difficult to be tagged in real-time by manual operation, which limits the detailed exploration of its neural mechanisms with the new techniques, such as fiber photometry recording or optogenetics. Here we introduced an arduino real-time jump tagging system (Art-JT system) to record the jump based on online monitoring the pressure changes of the floor in which the mouse is free exploring. Meanwhile, the Art-JT system can send the digital signal of the jump timing to the external device for tagging the events in the fiber photometry system or triggering the optogenetics laser. We tested it with the mice induced by Naloxone precipitated withdrawal jumping. The results showed that it could accurately record the jump events and provide several detailed parameters of the jump.

Furthermore, it was easy and fast to get the GCaMP6 signal correlated with the jump in the medial prefrontal cortex and primary motor cortex by combining the Art-JT system and multichannel fiber photometry system. Our results suggested that the Art-JT system may be a powerful tool for recording and analyzing jumping efficiently and helping us to understand stereotyped behavior.

\section{Introduction}

Jumping is one of the well-defined stereotyped behaviors in laboratory mice (1), which is repeated over and over again and seem to be without any apparent adaptive function (2). It can be found in the natural state of Bank voles and deer mice and disease models such as addiction and autism (3). Morphine and other prescription opioids are the main clinical treatments for moderate to severe acute pain. But in recent years, the abuse, dependence, and abuse of prescription opioids have become one of the most widely known public health problems. Morphine is a highly addictive opioid. Even with long-term abstinence, its recurrence rate is very high. The underlying neural mechanism of morphine addiction and recurrence is still elusive. Naloxone precipitated withdrawal model in mice is a commonly used screening model to evaluate the physical dependence of opioid analgesics (4). Increased jumping is typical behavior of withdrawal in mice (5-7). The current measurement of Naloxone precipitated withdrawal-induced jumps is limited to offline analysis with manual counting (5-7) or open source video analysis software (8). However, further research on jumping behavior requires real-time recording or regulation of the activities of specific brain regions and specific neuron types with new methods, such as GCaMP6 recording with fiber photometry and optogenetics.

In the current work, we introduced an Arduino microcontroller-based Art-JT system to record the jumping behavior through real-time monitoring of the pressure changes of the floor in which the mouse is free exploring. At the same time, the Art-JT system converted the timing of the jump to the transistor transistor logic (TTL) signal for triggering the external device. With the Art-JT system, we obtained several detailed parameters of the jump efficiently, such as jumping number, latency, speed, force. Combining with an ArtJT system and multichannel fiber photometry, we found that the neuron activities of the medial prefrontal 
cortex (mPFC) and primary motor cortex (M1) were highly correlated with Naloxone precipitated withdrawal jump and showed a different dynamic pattern in the GCaMP6 mice.

\section{Methods}

\section{Animals}

GCaMP6s male mice and wild-type C57BL/6J male mice at 8 weeks of age were purchased from the Experimental Animal Center of the Fourth Military Medical University (Xi'an, China). Then, mice were bred with a housing group of no more than 4 adults and all mice had free access to water and food within a 12-h light-dark cycle. All experiments were conducted during the light phase. Naloxone precipitated withdrawal test began after a week of handle. All procedures were approved by the Institutional Animal Care and Use Committee of the Fourth Military Medical University (FMMU) and carried out according to the "Principles of Medical Laboratory Animal Care" issued by the National Ministry of Health in China.

\section{Naloxone-precipitated withdrawal}

We used the C57BL/6J mice with naloxone-precipitated withdrawal jumping to test. Refer to the protocol in previous studies (10), mice were given morphine thrice daily (09:00 h, 13:00 h, and 17:00 h) for four days using a dosing schedule of 10,20, 40 and $40 \mathrm{mg} / \mathrm{kg}$ of morphine on days 1, 2, 3 and 4, respectively. On the last day (day 4), a final $40 \mathrm{mg} / \mathrm{kg}$ morphine dose was administered, followed by a single naloxone dose $(40 \mathrm{mg} / \mathrm{kg}) 3 \mathrm{~h}$ later. Immediately after naloxone injection, subjects were placed into Art-JT system to record the jumping frequency.

\section{Fiber photometry recordings}

Optical fiber $(230 \mu \mathrm{m}$ OD, $0.37 \mathrm{NA})$ was placed in a ceramic ferrule and inserted toward the mPFC (AP:1.95; ML:0.35; DV:-2.4) and M1 (AP:1.2; ML:1.75; DV:-1.35) in GCaMP6s mice. After 1 week for recovery, mice were used for naloxone-precipitated withdrawal and the fiber photometry system (QAXKFPS-MC-LED, Thinker Tech Nanjing Biotech Ltd, Nanjing, China) which connected with the Art-JT system was used for recording. The analog voltage signals were digitized at $500 \mathrm{~Hz}$ (Power 1401 digitizer, Cambridge Electronic Design) and sampled with software (TDMS, Thinker Tech Nanjing Biotech Ltd). Fiber-photometric recording data were exported as MATLAB files for further analysis. The fluorescence change values (dF/F\%) were calculated as (F-FO) / (FO-Voffset), where F0 is the baseline fluorescence signal averaged over a $2 \mathrm{~s}$ time-window prior to a trigger event and Voffset is the fluorescence signal recorded before the cannula was connected to the optical fiber. $\mathrm{dF} / \mathrm{F} \%$ values are presented as heatmaps or average plots with a shaded area indicating the SEM.

\section{Real-time recording and feedback of jumps with real-time closed-loop system}

After the injection of naloxone, the Arduino code was run, and a video was recorded on the computer. Then the mouse was put into the Art-JT system and was allowed to explore the cage freely for two 15 
min recordings. And there is a 5 min break between the two sessions. We used a 24-Bit HX711 pressure sensor (AVIA semiconductor, China) as the base of the Art-JT system's floor to automatically detect the body weight changes of the mouse caused by jumping and send the signal to an 8-bit MEGA2560 Rev3 microcontroller board (Arduino, Italy). The Arduino code can record the changes in body weight and used the principle of differential filtering to detect the waveform of the jump (Supplemental Code1). To remove the noise signal interference, the waveform is divided into peak and trough to compared. When the record is over, we can read the total number of jumps in the Arduino window. For the detailed parameters of jumping, such as the height and duration of each jump, we imported the data into MATLAB 2018a (Mathworks, USA) and ran the program (Supplemental Code2). In the meaning time, the activities of the mouse were videotaping from a side view with a USB webcam (Logitech HD Webcam C270). The timing of jumping was manually counted by trained observers with BORIS (version 7.9) (21). In the fiber photometry recordings test, Art-JT system was connected with fiber photometry system by BNC interface, TTL (5v) signal was passed to fiber photometry system when the mouse jumps into the air.

\section{Statistics}

All statistical analyses were performed in SPSS 21.0 (IBM, USA) and Origin Pro (OriginLab Corp., USA). All data were first analyzed by Mauchly's test of sphericity, and then analyzed the differences within the group by Repeated measures analysis of variance. Data were presented as mean \pm SEM. All error bars indicate SEM. In all cases, the criterion for statistical significance was $\mathrm{P}<0.05$.

\section{Results}

\section{The Art-JT system is a real-time pressure monitor controlled by an Arduino microcontroller}

The Art-JT system includes three parts: pressure sensor, Analog-to-digital converter (ADC), and Arduino microcontroller board (Fig. 1a). It can be connected with personal computer (PC) and fiber photometry system for online analysis and tagging the jump events. We selected a straingauge type transducer (Fig. 1a-1) as the pressure sensor since mice are lighter in weight and straingauge type transducer can achieve greater sensitivity, temperature compensation and eliminate the interference of non-measured directional stress. We put a chamber $(20 \mathrm{cmL} \times 20 \mathrm{cmW} \times 25 \mathrm{cmH})$ on top of the sensor for Naloxone-precipitated withdrawal jump test. The size of the chamber is based on previous studies to observe the withdrawal reaction in mice (9-12). Considering the matching with the sensor, we chose HX711 as the Analog-todigital converter (ADC) of this device (Fig. 1a-2). The operating voltage of the pressure sensor is $5-12 \mathrm{~V}$, which can be controlled by the Microcontroller Unit (MCU) direct power supply. The rated range $(r)$ is $3 K G$, and the measurement accuracy is Class $B(0.03 \% * r=0.1 \mathrm{~g})$, which fully meets the requirement of recording the activity of mice. The formula of the relationship between the output voltage U1 of the sensor and the pressure $\mathrm{F}$ and the reference voltage $\mathrm{UO}$ is as follows:

$\mathrm{U} 1=\mathrm{U} 0 * \mathrm{RO} * \mathrm{~F} / \mathrm{g} / \mathrm{r}$ 
(RO is the output of $2 \mathrm{mV} / \mathrm{V}, \mathrm{g}$ is the acceleration of gravity $9.8 \mathrm{~m} / \mathrm{s} 2, \mathrm{r}$ is the rated range of $3 \mathrm{Kg}$, and the estimated value range of $\mathrm{U} 1$ is $0-10 \mathrm{mV}$.)

The last and most important part is Arduino microcontroller board (Fig. 1a-3). The following is the working principle of Art-JT system: it converts the pressure signal into a voltage signal in the cage, and then converts the voltage signal into a digital signal in $A D C$, and then Arduino records and judges the signal, finally output at the PC and send the TTL signal to fiber photometry system for marking every jump (Fig. 1b). As Ardiuno code (Supplemental Code1) principle showing in the flowing chart in Fig. $1 \mathrm{c}$, the recording begins when " $\mathrm{R}$ " is entered. The signals will be updated 10 times per second. Meanwhile, Arduino used the principle of differential filtering to detect the waveform of the jump, when the jump is confirmed, in addition to being recorded by the Ardiuno, TTL signal was sent to the fiber photometry system at the same time. The recording stops when " $X$ " is entered.

\section{The Art-JT system could record the whole process of jump and provide detailed parameters of the jump}

We first tested whether Art-JT system could detect the jump of the mouse in it. Fig. 2a showed a representative trace of the pressure changes during the mouse exploring in the chamber. The positive peak means the pressure decreasing which is caused by the mouse's four legs or forelimbs leaving the cage board. The negative peak means the pressure increasing which usually is caused by the mouse squatting on the board and preparing to jump. Combining with video and recorded pressure trace, we found three types of the jump. The first one is direct jump (Fig. 2a-1), the mouse just jumped to air directly without any preparation, so we can only see a single positive peak or tiny negative peak followed by a big positive peak. The second type is fake jump (Fig. 2a-2), we can only see a negative peak with this type. The mouse just squatted with its hind legs and was preparing to jump. But somehow the mouse did not leave the floor. For the above two situations, algorithmic improvements are made to the code. Finally, only the classic complete jump was included for further analysis. It could be divided into four stages (Fig. 2a3): Stage 1 is that the mouse just walking or resting on the floor of the cage. It's four legs all on the board. At this time, the recording trace was showing the real body weight of this mouse. Stage 2 is that the mouse was preparing to jump and squatted with its hind legs. The trace showed a huge negative peak. The amplitude of the peak could increase one third or one-half of the body weight. Stage 3 is that the mouse was hanging in the air or on the top beam of the cage. The trace reached 0 . Stage 4 is that the mouse fell back to the floor. The accelerated velocity produced a big negative peak on the trace. The value could be similar or even bigger than stage 2 .

Based on the accurate record of the jumping timing, the body weight of the mouse getting from the Art-JT system, we were wondering whether we could get detailed information from this device. We wrote the codes in Matlab (Supplemental Code 2) to run the further analysis and get various parameters. It included the body weight when the mouse was preparing to jump, squatting force with its hind legs, the latency of the jump, the speed at which the mouse jumps (Velocity) in stage2, the height of the jump, and the number of jumps $(\mathrm{N})$ of the mouse (Fig. 2b). We could evaluate the whole view of the jump from these parameters. For example, the squatting force might reflect muscle strength and also motor system 
function. The latency and the speed of jumps might demonstrate the level of Naloxone precipitated withdrawal. Besides that, we could get the frequency distribution of the inter-jumping interval (Fig. 2c) and the precise inter-jumping interval during the time course (Fig. 2d) which illustrated the dynamic characteristics of stereotyped jump behavior. With these parameters, we could get more information on jumping behavior which may be helpful to understand its neural mechanisms in the future.

\section{The Art-JT system has accurate number counting and timing recording function for the jump}

Although Art-JT system can easily and quickly get the total number of mouse jumps and rich parameters about each jump, we still need to confirm that the jumps we recorded indeed correspond to the real jumps of the mice. So, we compared the data of jumping timing and numbers between observers and our device. Fig. 3a demonstrated the whole traces of the jumping behavior from our device and manual count from one mouse. The top trace is the pressure change curve getting from Art-JT system. The arrow means the timing of a tested mouse putting in the cage. The value increased from 0 to the body weight of the tested mouse. A series of peaks are the jumping behavior of the tested mouse during the whole recording period. The below trace is a timeline getting from manually counting. Both traces showed a similar pattern and also a strong correlation. Then we compared the total number of the jump getting from our system and two independent observers. There is no significant difference between Art-JT system and two observers in the total number of jumps (Repeated ANOVA, $F=2.406, p>0.05$; Fig. 3b). We also examined the jumping number difference between either machine and observer or observer themselves. We found the value of the number difference between observers seems greater than it between the machine and observer, although the statistics did not show the significant difference (One way ANOVA, F = 2.406, $p>0.05$; Fig. $3 c$ ). By comparing the timing of the jump from manual counting to machine counting, we also noticed that the timing of the jump from manual counting gradually developed a delay compared to machine counting. And the delay time even increased gradually (Fig. 3d) as recording time went by, which may be caused by the tired observer.

\section{The Art-JT system could send the jumping timing to the fiber photometry system for tagging the $\mathrm{Ca}^{2+}$ signal related to the jump}

Recently, genetically encoded calcium indicator (GECl), such as GCaMP6, was used for exploring the relationship between the certain neuron populations activities and the certain behavior through in vivo fiber photometry recording. In this method, accurate timing of the behavioral events is important for analyzing the Calcium dynamics correlated the behavior. The Art-JT system could convert the real timing of the jump events to 5V TTL signal and send it to the fiber photometry system as the event tag. To test this possibility, we implanted the optical fiber in the MPFC and M1 (Fig. 4a) in GCaMP6s mice and induced these mice to generate Naloxone-precipitated withdrawal jump. The results showed that the fluctuation of the $\mathrm{Ca}^{2+}$ signal recorded by the fiber photometry system and the received TTL signal correspond to each other on the time axis in MPFC (Fig. 4b). The $\mathrm{Ca}^{2+}$ signal changes in each jump were successfully recorded in these two brain regions and superimposed into a $\mathrm{Ca}^{2+}$ signal change curve with a small standard deviation (Fig. 4c). But the pattern of $\mathrm{Ca}^{2+}$ activity caused by the jump in the two brain 
regions seems to be different. The $\mathrm{Ca}^{2+}$ activity in $\mathrm{mPFC}$ quickly returned to baseline after the jumping, while the calcium activity in M1 even was lower than the baseline after the jump event stop. And the latency of the peak of $\mathrm{Ca}^{2+}$ activity in the two brain regions also showed some difference. But statistical analysis was not performed due to the small sample size (Fig. 4d). This implies there may be an order in which Naloxone-precipitated withdrawal jumps activate each brain region. Moreover, we found there is a difference in the area under the curve (AUC) of $\mathrm{Ca}^{2+}$ activity between $\mathrm{M} 1$ and mPFC between $0-1$ seconds $(p<0.001)$ (Fig. 4e). It suggested that the neuron activities of mPFC and M1 were different in Naloxoneprecipitated withdrawal jumps. And our Art-JT system is a powerful tool for exploring the neural mechanism of stereotyped jump behavior.

\section{Discussion}

In the current study, we designed and built a device named Art-JT system which could be used for automatically detecting the stereotyped jumping behavior and marking jump events in fiber photometry in real time. Through the conversion of pressure signal - voltage signal - digital signal, Art-JT system used pressure sensor and Arduino to quickly and accurately make real-time judgments for every jump based on pressure changes and send TTL signal to fiber photometry system for the recording of every jump in real time. Based on the data getting from Art-JT system, we can not only obtain the total jumping number fast and accurately but also get more detailed parameters of jumping behavior.

Animal behavior is important for helping us to understand the working operation of the central neural system. However, many animal behaviors still rely on manual analysis, which is very time-consuming and inaccurate. Recently, the combination of Arduino microcontroller and behavior characteristics capturing to flexibly record and judge behaviors is becoming popular because of its simplicity and speed, which gradually replace the laborious manual analysis of behaviors $(13,14)$. Compared with manual counting, Art-JT system not only saves time and energy but also improves the accuracy of jumping records. More importantly, it breaks the limitations of neural activity records and regulation of behaviors that occur within a very short period of time, such as jumping. According to the Art-JT system, the jumping duration of mice is about $400 \mathrm{~ms}$, which means that observers who have a 300-400 ms response in the best state cannot accurately record the moment when the jump occurs. But Art-JT system with higher resolution can not only record exactly when the jump occurs but also can send the signal to an external device either for tagging the event in the fiber photometry recording or triggering laser to do optogenetics stimulation. Besides that, Art-JT system also offers more detailed parameters about jumping for us, those parameters provide more possibilities for further research on the neural mechanism of jumping behavior. Some video analysis software with the machine-learning method can also analyze the jumping behavior and provide parameters in real-time. However, the accuracy of the detecting is highly relied on the training and may produce variability from the lab to the lab. The principle of our Art-JT system is simple and straightforward. We think it will increase repeatability between different labs.

The study of abnormal animal behavior has played a huge role in solving many human diseases, the research methods of animal behavior are constantly improving and updating to meet the needs. On the 
behavioral phenotype of stereotyped jumping behaviors in mice, we introduce the Art-JT system for more efficient and accurate recording. In addition to the naloxone precipitated withdrawal test used to check the Art-JT system in the article, Art-JT system can also be applied to other animal models. Some of the autism model mice also exhibited repeated jumping behaviors, such as C58 (15) and Shank2 ${ }^{-/-}$mice (11, 16), and some obsessive-compulsive disorder (OCD) model mice have stereotypic jump, such as the deer mice (17). Parameters from Art-JT system can provide rich data about muscle ability and stereotyped behavior, it may provide new ideas for muscle assessment after stroking (18) and repetitive behaviors in autism model mice $(19)$ or OCD mouse model $(17,20)$.

Therefore, our Art-JT system can not only record exactly when the jump occurs but also can send the signal to an external device either for tagging the event or triggering laser to do optogenetics stimulation. It can provide better automatic recording and precise online regulation methods for the study of the intrinsic neural mechanisms of stereotyped behavior and related disease models.

\section{Abbreviations}

Art-JT system: arduino real-time jump tagging system

ADC: Analog-to-digital converter

MCU: Microcontroller Unit

PC: Personal compute

TTL: Transistor transistor logic

mPFC: Medial Prefrontal Cortex

M1: Primary Motor Cortex

AUC: Area under the curve

\section{Declarations}

Ethics approval and consent to participate: Not applicable.

Consent for publication: Not applicable.

Availability of data and materials: The datasets used and/or analysed during the current study are available from the corresponding author on reasonable request.

Competing interests: The authors declare that they have no competing interests. 
Funding: This work was supported by Natural Science Foundation of China (81771476 and 82071536 to Wenting Wang, 81730035 to Shengxi Wu), CAS Key Laboratory of Brain Connectome and Manipulation (2019DP173024 to Wenting Wang) and Shaanxi Key Research and Development Program (Grant No. 2020SF-127 to Qian Xue).

Authors' contributions: W.W. and Y.L. designed and supervised the study. J.G. and and G.L. performed in design and establishment of the device. H.Z. is responsible for the mouse withdrawal model. J.G., operated the surgery, behavior test, and statistical analysis. C.Q., H.L., and Y.L., analyzed and interpreted data. W.W., J.G. and Q.X. prepared graphs, wrote, and revised the manuscript. All authors reviewed, revised, and approved the manuscript for publication.

Acknowledgements: Not applicable.

\section{References}

1. Mason G, Rushen J. Stereotypic animal behaviour: Fundamentals and applications to welfare. Wallingford: CABl; 2006.

2. Ellenbroek BA, Cools AR. Chapter 20 - Stereotyped behaviour. In: van Haaren F, editor. Techniques in the Behavioral and Neural Sciences. 10: Elsevier; 1993. pp. 519-38.

3. Won H, Lee HR, Gee HY, Mah W, Kim JI, Lee J, et al. Autistic-like social behaviour in Shank2-mutant mice improved by restoring NMDA receptor function. Nature. 2012;486(7402):261-5.

4. Dunn KE, Huhn AS, Bergeria CL, Gipson CD, Weerts EM. Non-Opioid Neurotransmitter Systems that Contribute to the Opioid Withdrawal Syndrome: A Review of Preclinical and Human Evidence. J Pharmacol Exp Ther. 2019;371(2):422-52.

5. Pang G, Wu X, Tao X, Mao R, Liu X, Zhang YM, et al. Blockade of Serotonin 5-HT2A Receptors Suppresses Behavioral Sensitization and Naloxone-Precipitated Withdrawal Symptoms in MorphineTreated Mice. Front Pharmacol. 2016;7:514.

6. Sharf R, Sarhan M, Dileone RJ. Orexin mediates the expression of precipitated morphine withdrawal and concurrent activation of the nucleus accumbens shell. Biol Psychiatry. 2008;64(3):175-83.

7. Wu X, Pang G, Zhang YM, Li G, Xu S, Dong L, et al. Activation of serotonin 5-HT(2C) receptor suppresses behavioral sensitization and naloxone-precipitated withdrawal symptoms in herointreated mice. Neurosci Lett. 2015;607:23-8.

8. Adamah-Biassi EB, Stepien I, Hudson RL, Dubocovich ML. Automated video analysis system reveals distinct diurnal behaviors in C57BL/ 6 and C3H/HeN mice. Behav Brain Res. 2013;243:306-12.

9. el-Kadi AO, Sharif SI. The influence of various experimental conditions on the expression of naloxone-induced withdrawal symptoms in mice. Gen Pharmacol. 1994;25(7):1505-10.

10. Kest B, Palmese CA, Hopkins E, Adler M, Juni A, Mogil JS. Naloxone-precipitated withdrawal jumping in 11 inbred mouse strains: evidence for common genetic mechanisms in acute and chronic morphine physical dependence. Neuroscience. 2002;115(2):463-9. 
11. Kim H, Lim CS, Kaang BK. Neuronal mechanisms and circuits underlying repetitive behaviors in mouse models of autism spectrum disorder. Behavioral brain functions: BBF. 2016;12(1):3.

12. Sharif SI, el-Kadi AO. The role of cholinergic systems in the expression of morphine withdrawal. Neuroscience research. 1996;25(2):155-60.

13. D'Ausilio A. Arduino: a low-cost multipurpose lab equipment. Behavior research methods. 2012;44(2):305-13.

14. Devarakonda K, Nguyen KP, Kravitz AV. ROBucket: A low cost operant chamber based on the Arduino microcontroller. Behavior research methods. 2016;48(2):503-9.

15. Ryan BC, Young NB, Crawley JN, Bodfish JW, Moy SS. Social deficits, stereotypy and early emergence of repetitive behavior in the C58/J inbred mouse strain. Behav Brain Res. 2010;208(1):178-88.

16. Schmeisser MJ, Ey E, Wegener S, Bockmann J, Stempel AV, Kuebler A, et al. Autistic-like behaviours and hyperactivity in mice lacking ProSAP1/Shank2. Nature. 2012;486(7402):256-60.

17. Korff S, Stein DJ, Harvey BH. Stereotypic behaviour in the deer mouse: pharmacological validation and relevance for obsessive compulsive disorder. Prog Neuro-psychopharmacol Biol Psychiatry. 2008;32(2):348-55.

18. Mittal N, Pan J, Palmateer J, Martin L, Pandya A, Kumar S, et al. So you think you can jump? A novel long jump assessment to detect deficits in stroked mice. J Neurosci Methods. 2015;256:212-9.

19. Wang S, Tan N, Zhu X, Yao M, Wang Y, Zhang X, et al. Sh3rf2 Haploinsufficiency Leads to Unilateral Neuronal Development Deficits and Autistic-Like Behaviors in Mice. Cell reports. 2018;25(11):296371. e6.

20. Wolmarans de W, Brand L, Stein DJ, Harvey BH. Reappraisal of spontaneous stereotypy in the deer mouse as an animal model of obsessive-compulsive disorder (OCD): response to escitalopram treatment and basal serotonin transporter (SERT) density. Behav Brain Res. 2013;256:545-53.

21. Friard O, Gamba M. BORIS: a free, versatile open-source event-logging software for video/audio coding and live observations. 2016;7(11):1325-30.

\section{Supplemental Codes}

The Supplemental Codes 1 and 2 are not available with this version.

\section{Figures}




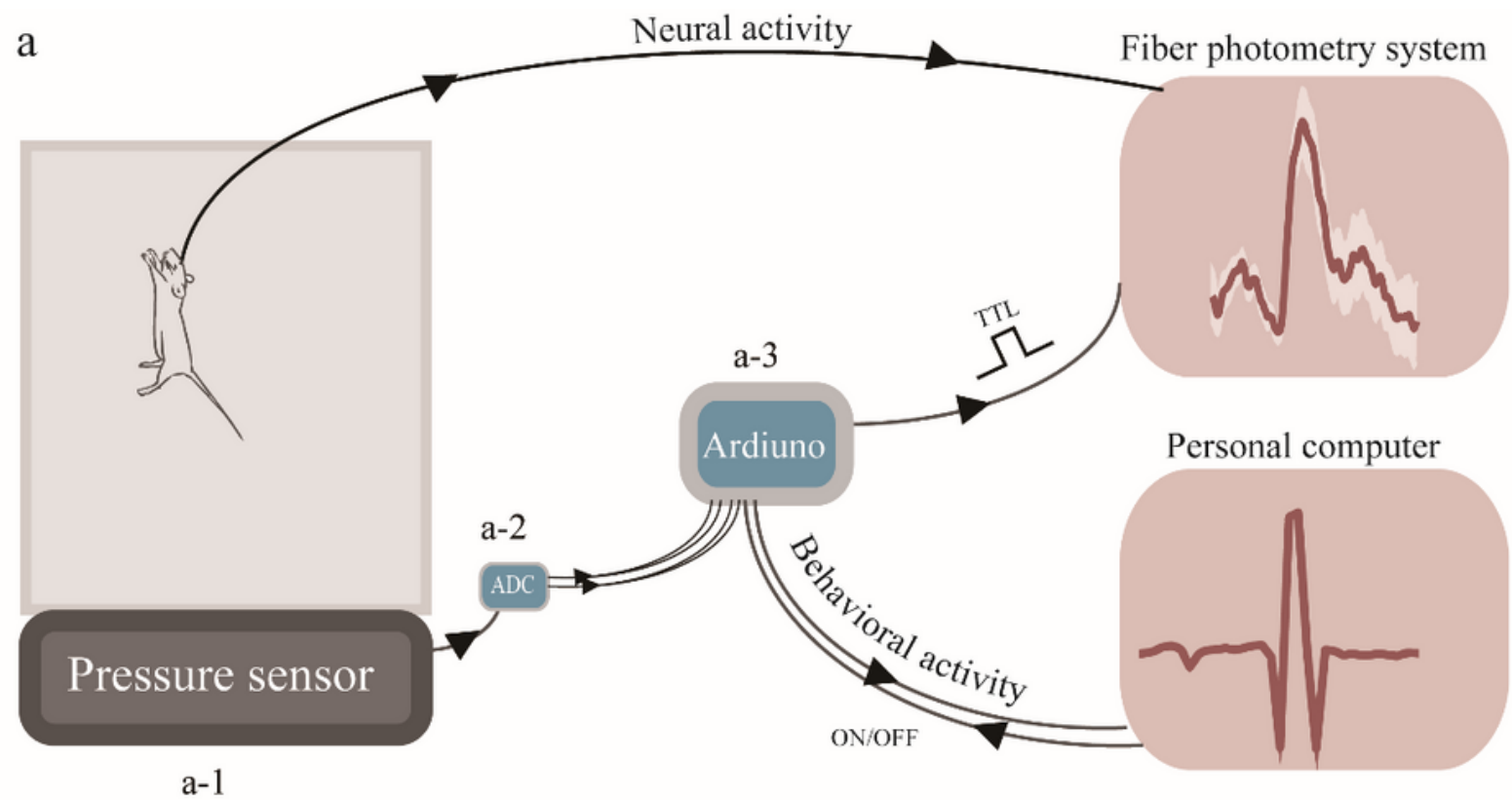

b

$\mathrm{c}$

Pressure signal from the activity of mice

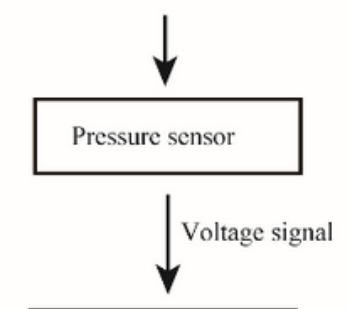

Analogue-to-digital conversion (ADC)
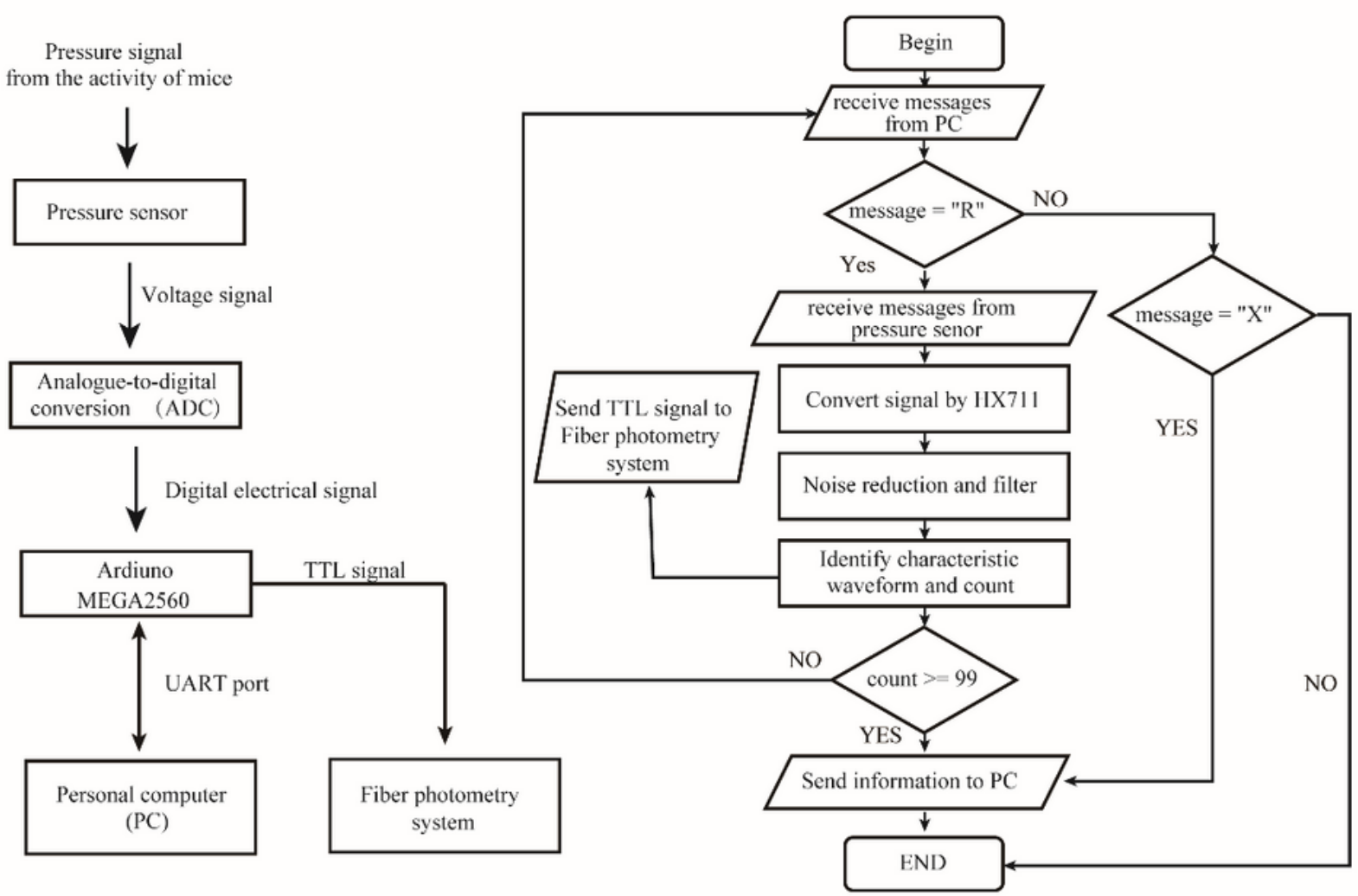

\section{Figure 1}

The basic components and principle of Art-JT system (a) Art-JT system includes the following parts: the pressure sensor ( $a-1)$ under the floor of the cage, an Arduino board (a-3) with Analogue-to-digital conversion (ADC) (a-2) for the conversion of signal. (b) Art-JT system convert the pressure signal into voltage signal by sensor, and then convert the voltage signal into digital signal in $A D C$, finally Arduino records and judges the signal, finally output at the PC or send TTL signal to Fiber photometry system. (c) 
Arduino record and judge jumping by the code, recording begins when " $\mathrm{R}$ " is entered, meanwhile, Arduino used the principle of differential filtering to detect the waveform of jump, recording stops when " $X$ " is entered.

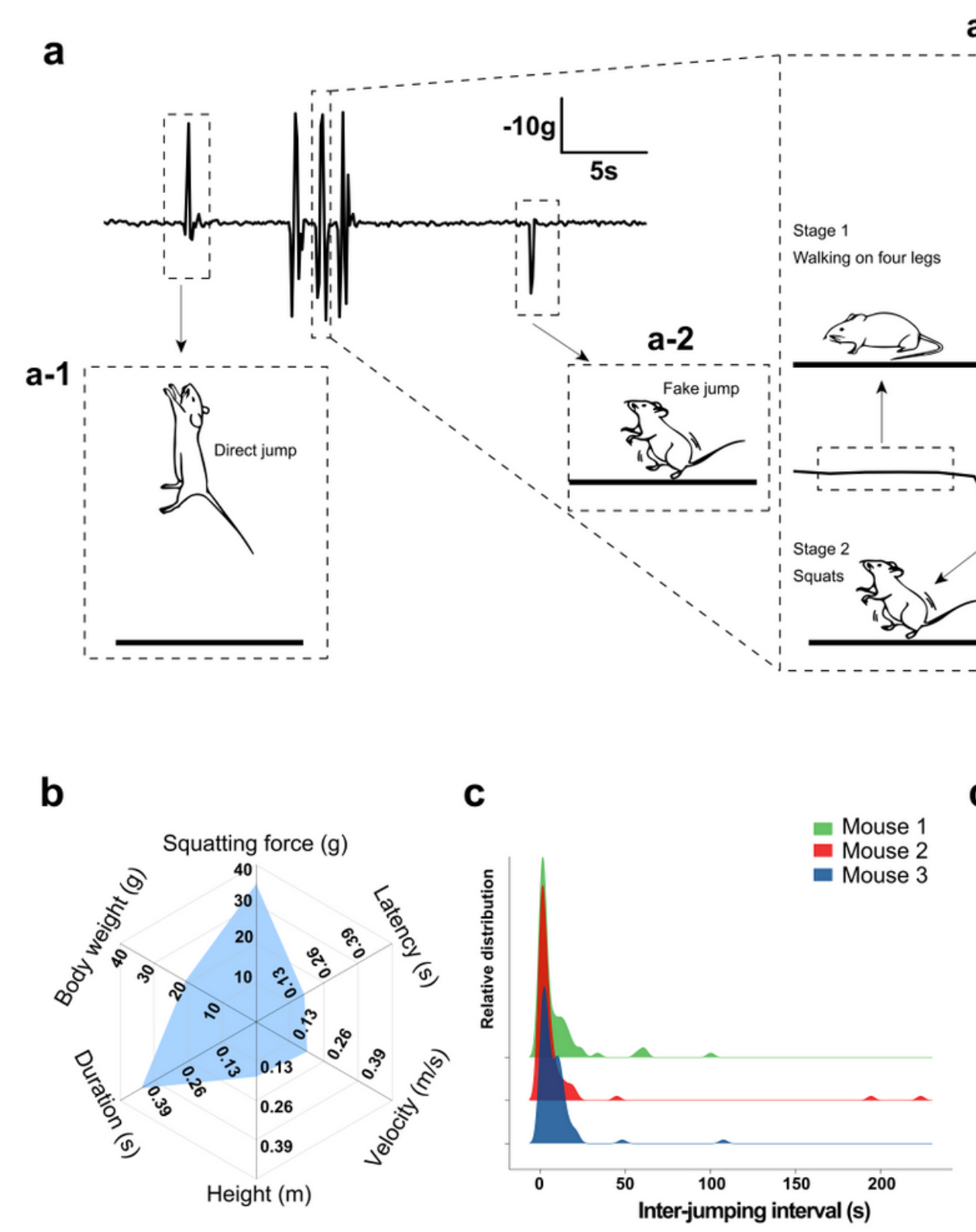

a-3 Complete jump

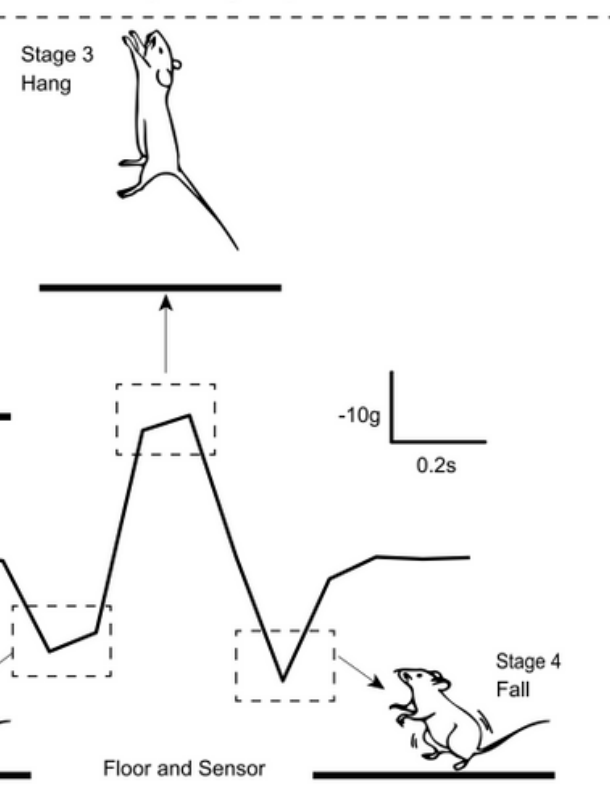

d

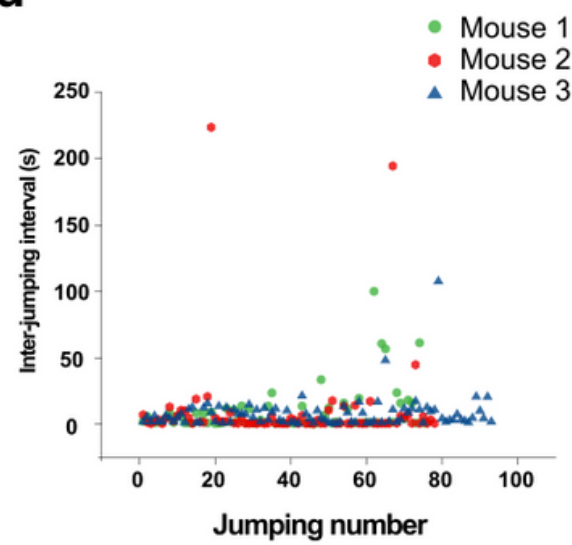

Figure 2

The whole process and the detail parameters of the jump provided by the Art-JT system (a) A representative trace of Jumping waves based data from Art-JT system. It includes three types: direct jump without squats and fall stage (a-1), fake jump just has squats and no real jumping (a-2), and the complete jump we focus on (a-3). Complete jump consists of four stages. Stage 1 is that the mouse just walking or resting on the floor of the cage. Stage 2 is that the mouse was preparing to jump and squatted with its hind legs. The trace showed a huge negative peak. Stage 3 is that the mouse was hanging in the air or on the top beam of the cage. The trace reached to 0 . Stage 4 is that the mouse fell back the floor. (b) A radar map showed the intact view of the jumping behavior combined several degrees, including 
squatting force, jump latency, jump speed, jump height, jump duration, and body weight of the mouse. (c) The frequency distribution of inter-jumping intervals from three example mice showed a similar relatively high frequency of the jumping. (d) The inter-jumping interval with the time course demonstrated these three mice kept jumping with high frequency during the whole recording time.

a

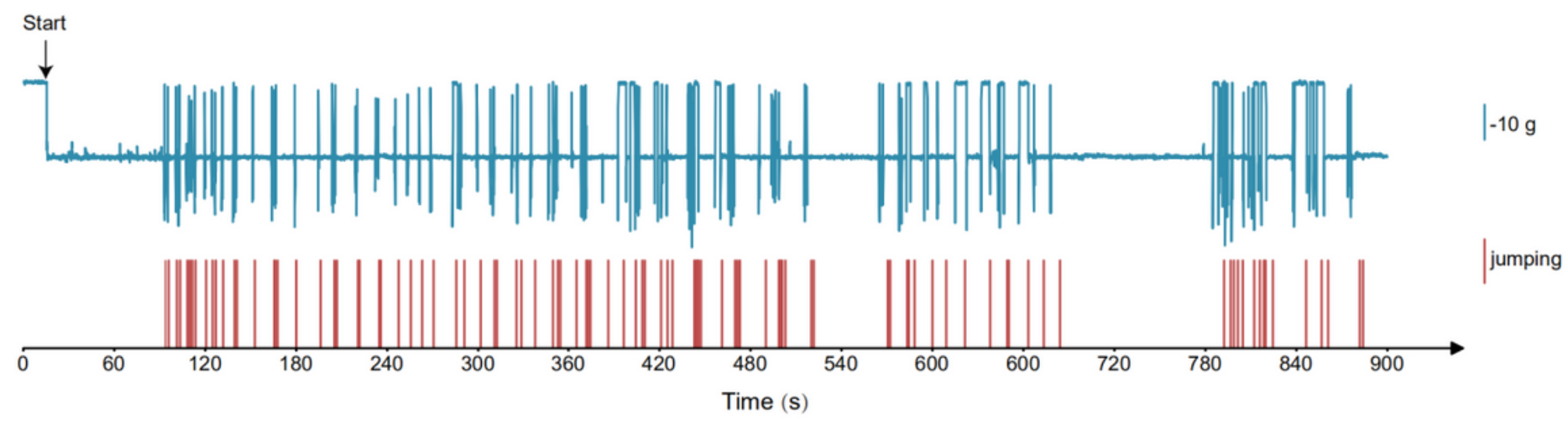

b

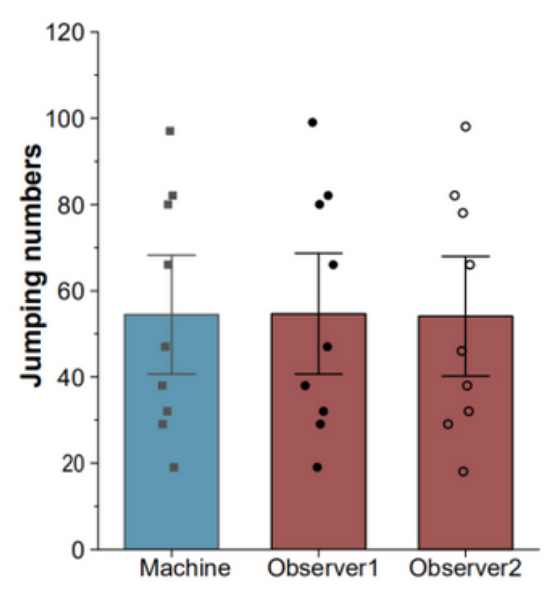

c

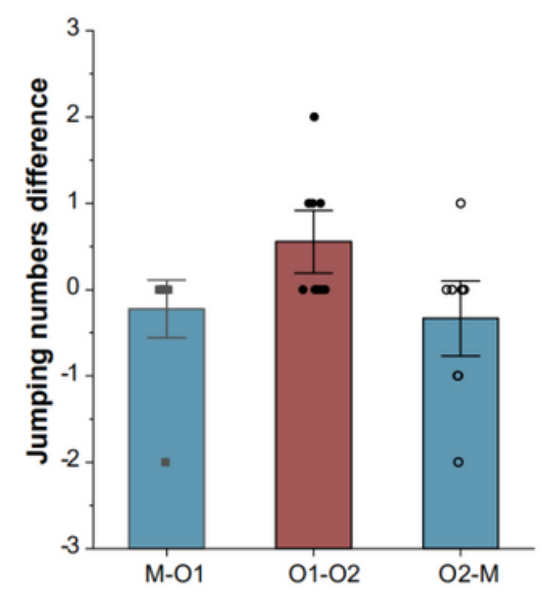

d

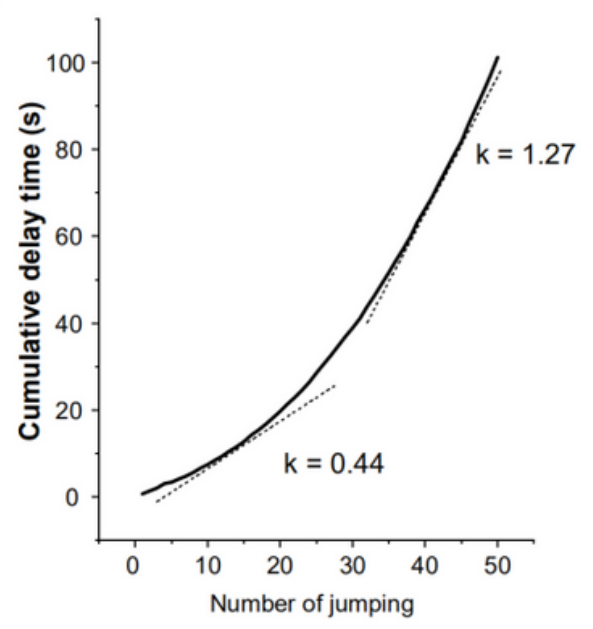

Figure 3

The Art-JT system detected more accurate jump compared with manual observers (a) A one-to-one correspondence between manual count and Art-JT system count on the same timeline. (b) No significance between Art-JT system and two observers in total number of jumps ( $F=2.406, P>0.05)$. (c) Although there is no statistical difference here $(F=2.406, P>0.05)$, variability between observers was greater than variability that between observer and Art-JT system. (d) The delay of the observer's count increases with time. 

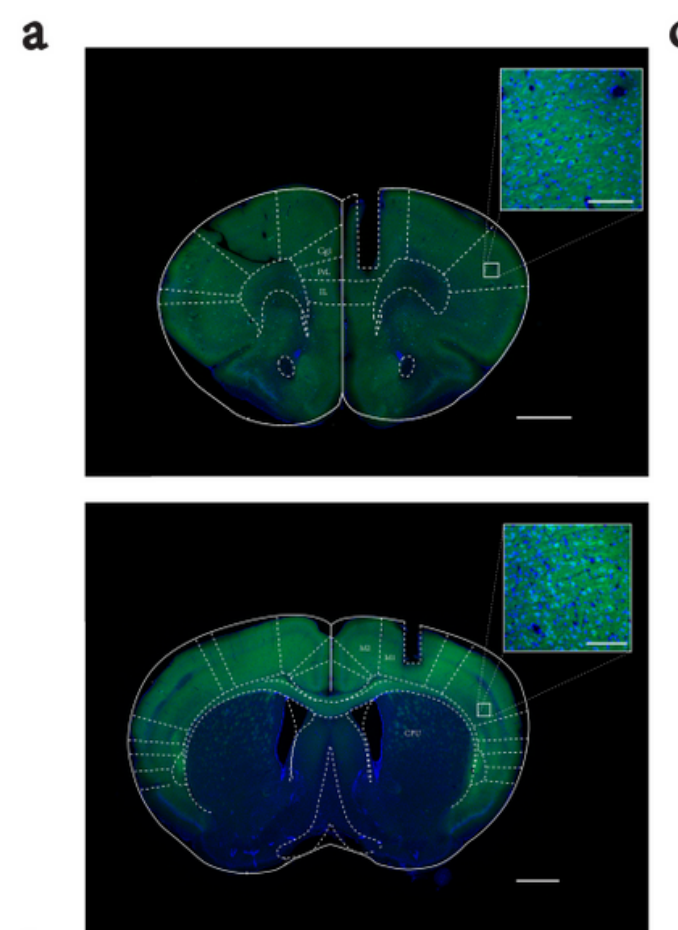

b
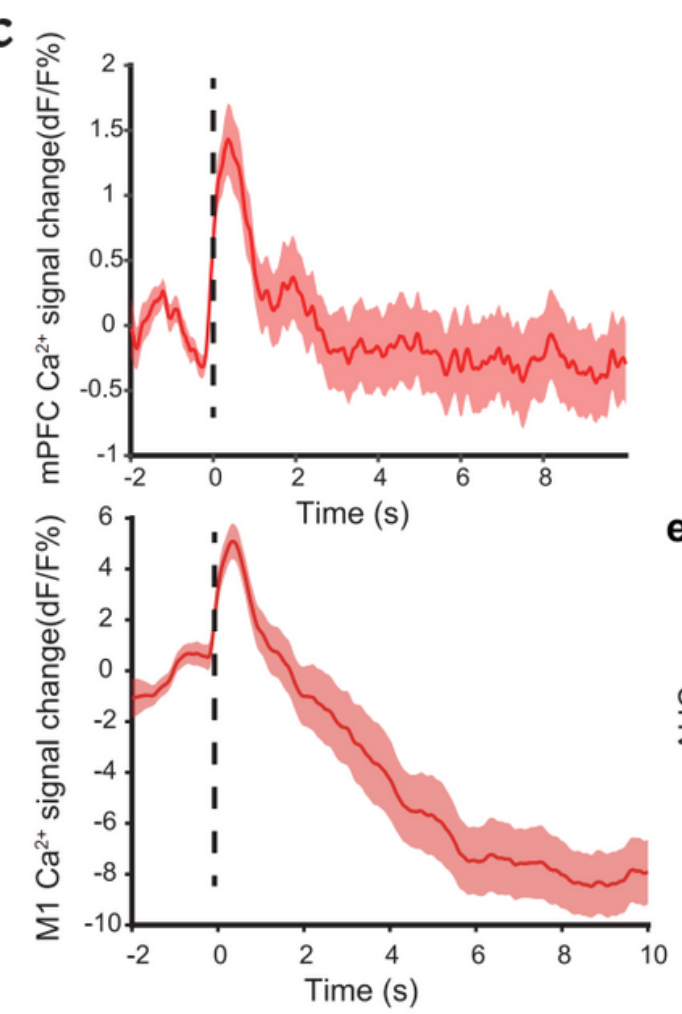

d
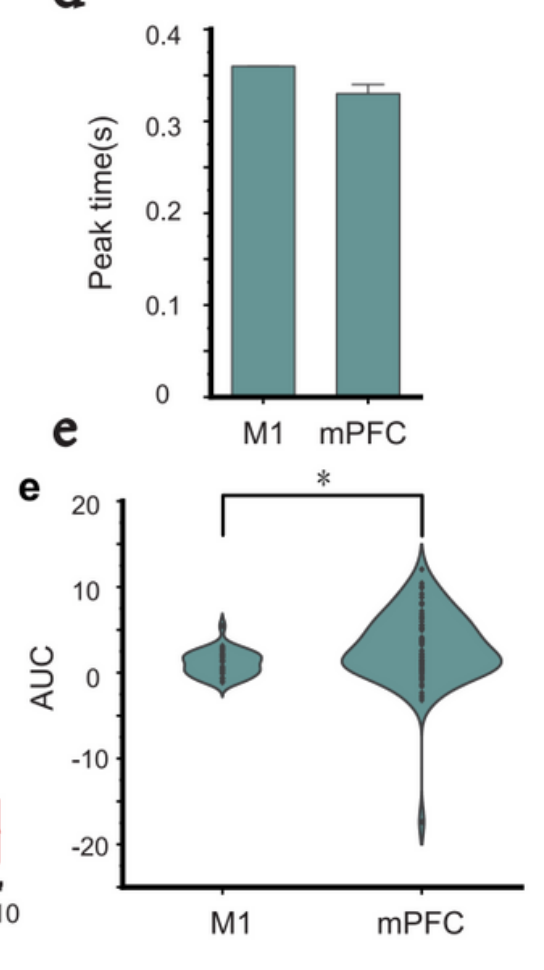
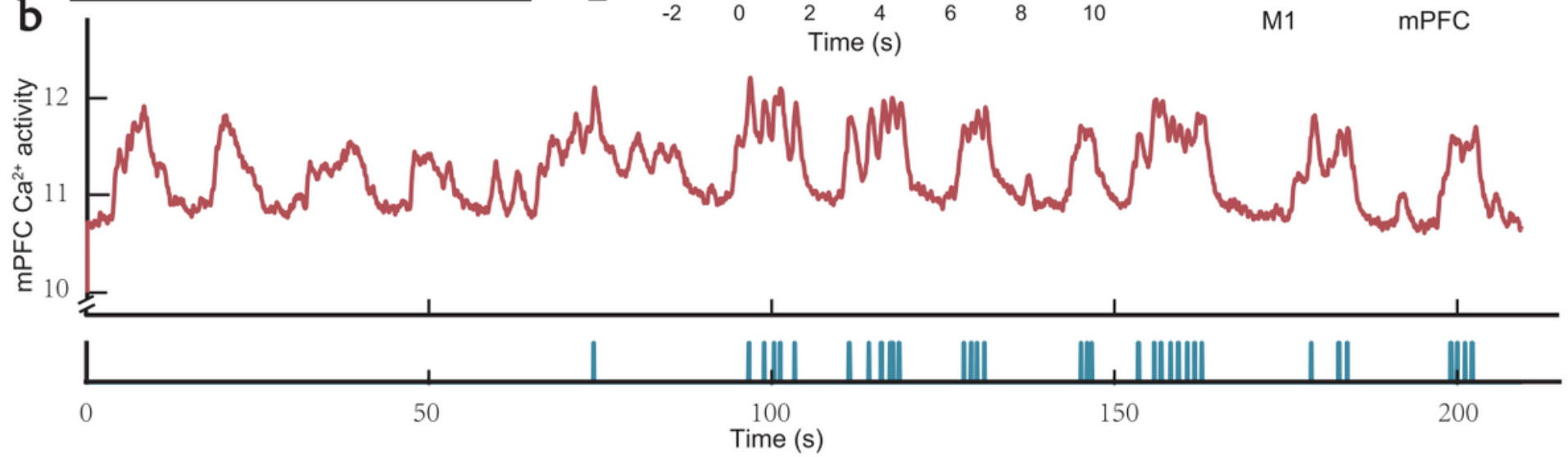

Figure 4

The Art-JT system provided the precise timing of the jump for the GCaMP 6 signal (a) The overlay of

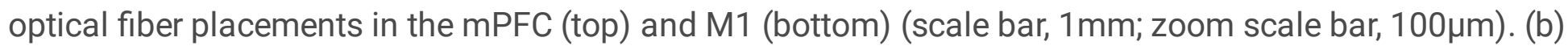
The overall correspondence between calcium activity and the TTL signal output by the Art-JT system for each jump. (c) \%dF/F of calcium activity signals in mPFC (top) and M1 (bottom). (d) The difference in the time point when calcium activity reaches its peak between mPFC and M1. (e) There is a difference in the area under the curve of Ca2+ activity between M1 and mPFC between 0-1 seconds $(F=6.76, p<0.05)$. 